Please do not remove this page

RMIT

UNIVERSITY

\title{
Quadrature hybrid coupler using photonic transversal approach
}

Emami, Hossein; Bui, Lam; Mansoori, Sana; Mitchell, Arnan

https://researchrepository.rmit.edu.au/esploro/outputs/9921862659201341/filesAndLinks?institution=61RMIT_INST\&index=null

Emami, H., Bui, L., Mansoori, S., \& Mitchell, A. (2006). Quadrature hybrid coupler using photonic transversal approach. Proceedings of the 31st Australian Conference on Optical Fibre Technology and Australian Optical Society (ACOFT\&AOS 2006). https://doi.org/10.1109/ACOFT.2006.4519214

Published Version: https://doi.org/10.1109/ACOFT.2006.4519214

Repository homepage: https://researchrepository.rmit.edu.au (c) 2006 IEEE. Personal use of this material is permitted. However, permission to reprint/republish this material for advertising or promotional purposes or for creating new collective works for resale or redistribution to servers or lists, or to reuse any copyrighted component of this work in other works must be obtained from the IEEE.

Downloaded On 2023/04/26 20:18:26 +1000 


\title{
Quadrature Hybrid Coupler Using Photonic Transversal Approach
}

\author{
Hossein Emami ${ }^{1}$, Lam Anh Bui ${ }^{1}$, Sana Mansoori ${ }^{2}$ and Arnan Mitchell ${ }^{1}$ \\ ${ }^{1}$ Microelectronics and Materials Technology Centre, School of Electrical and Computer Engineering, \\ RMIT University, GPO Box 2476V, Melbourne, Victoria 3001, Australia
}

${ }^{2}$ Department of Electrical and Electronics Engineering, College of Engineering, University of Bahrain

\begin{abstract}
A novel technique to phase shift signal by 90 degrees over a broad frequency range based on transversal signal processing is proposed and practically demonstrated. The proposed technique is suitable for non coherent optic implementation.
\end{abstract}

\section{INTRODUCTION}

A Quadrature hybrid is a device that splits an RF signal into two equal components and imparts a $90^{\circ}$ phase shift onto one of the outputs with respect to the other. These devices are one of the basic components used in a variety of microwave circuits such as balance mixers, data modulators [1], phase shifters, and beam formers [2]. It is important that these devices provide a low-loss, equal power split between the two output ports and maintains a precisely $90^{\circ}$ phase shift over multiple octaves.

Broadband quadrature hybrid coupler implementations using traditional microwave techniques have been reported [1-4]. Practical devices have moderate bandwidths $(2-18 \mathrm{GHz})$, but exhibit significant amplitude imbalance $(>3 \mathrm{~dB})$. Impedance matching presents a significant challenge that must be overcome using multiresonant structures. These structures have very stringent fabrication tollerences and may even need trimming and can thus be very expensive $[3,4]$.

Modern electronic warfare systems are being considered to cover an extended range from $2-40 \mathrm{GHz}$ and beyond. Traditional microwave techniques may not be capable of realizing quardature hybrids that cover this extremely broad bandwidth and still maintain acceptable amplitude balance, phase response and practical fabrication tolerances.

In recent years, microwave photonics has been investigated as a means of achieving ultra-broadband microwave signal processing. Indeed, several microwave photonic quadrature hybrid implementations have been proposed $[5,6]$. These solutions employ coherent optical beating to transfer the optical phase into electrical phase [5]. Unfortunately, these systems rely on the maintenance of optical coherence throughout the system and thus may not be robust when used in a harsh defence environment.

Another microwave photonic technique that has been introduced recently is the photonic transversal filter [7]. Here, the RF signal is modulated onto several optical carriers (or taps) and each tap is delayed by a different amount before detection. By controlling the relative weights and delays of each tap, a number of microwave signal processing operations can be realized $[7,8]$.
We have recently proposed the use of a microwave photonic transversal filter to realize a broadband, highperformance transversal filter [9]. This paper presenta a practical implementation of this concept using a simple four tap transversal filter. A microwave photonic hybrid with comparable performance to traditional microwave implementation is demonstrated and paths for extension to ultra-broad bandwidths are discussed.

\section{THEORY}

As discussed in [9], transversal filtering is a method by which a range of signal processing functions can be achieved. In short, a signal is divided between a number of taps and each tap is delayed by a different amount. At the output, all of the delayed taps are summed and detected. By controlling the relative weights and delays of each tap, a range of filter functions can be implemented.

Each tap in the transversal filter can be considered as a separate sample in the time domain with each delayed tap corresponding to a different point in time. By adjusting the amplitude of each tap, an arbitrary impulse response can be formed. The frequency response of the summation of these delayed taps will be the Fourier transform of the impulse response. Careful control the tap amplitudes enables a broad range of filter functions to be realized.

It is well known that if a system has an impulse response of a homographic hyperbola type, the corresponding frequency response will have a constant amplitude and a frequency independent $90^{\circ}$ degree phase shift. The impulse response of such a Hyperbola is presented in Figure 1 and the corresponding frequency response is presented in Figure 2.

For ideal system performance with infinite bandwidth, the impulse response has to be an infinite continuous time signal. In reality, this is impractical, and thus the impulse response must be truncated in time. Windowing techniques are usually employed to reduce distortions of the system frequency response due to Gibes effect [9].

The impulse response in transversal filter is sampled by discrete taps which are effectively delta functions in time. Thus, along with being truncated, the impulse response is samples as shown in Fig 1. Sufficient samples must be included to avoid aliasing distortion. Ideally, appropriate windowing should enable synthesis of a practical filter with performance similar to its continuous counterpart. Fig 2 presents the predicted amplitude and phase of the frequency response of both the continuous, infinite and also the sampled and truncated responses shown in Fig 1. 


\section{METHOD}

To implement the transversal filter depicted in Figs 1 and 2, we have chosen to use a wavelength division multiplexing (WDM) photonic technique. Figure 3 presents a diagram of the experimental set up.

To demonstrate the concept of photonic RF hybrid coupler. Four lasers with different wavelength have been used to implement a four taps transversal filter using wavelength division multiplexing (WDM). Each laser goes through a polarization controller (PC). Two laser beams then are combined by $3-\mathrm{dB}$ couplers going through Mach-Zehnder modulators biased at $\mathrm{V}^{+}$and $\mathrm{V}^{-}$to experience a 180 degree phase difference respect to each other. The outputs of the MZMs are then goes through Variable Optical Lengths (VOL) to make sure that both optical links have the same lengths.

The two outputs are then combined and enter a length of dispersive fibre. This fibre provides a different delay for each wavelength. The dispersed signals are then amplified by an Erbium Doped Fiber Amplifier (EDFA). The output of EDFA is then split into two portions by a $10 \%$ optical coupler. The $10 \%$ portion goes to an Optical Spectrum Analyzer (OSA) to monitor the amplitude of each tap and the $90 \%$ portion goes to a Photo Detector (PD) which is connected to the Port II of a $20 \mathrm{GHz}$ Vector Network analyzer (VNA), while port I is connected to an RF power splitter feeding RF inputs of MZMs.

For $20 \mathrm{GHz}$ bandwidth, the spacing of the samples of the impulse response must be $\Delta t=51.23$. From this tap spacing it is possible to work out what wavelength spacing is required. Using $\Delta t=\mathrm{D} \Delta \lambda \mathrm{L}$, with dispersion constant $\mathrm{D}=17 \mathrm{ps} / \mathrm{nm} . \mathrm{km}$, dispersive fibre length $\mathrm{L}=2.354 \mathrm{~km}$, the wavelength separation is found to be $\Delta \lambda=1.25 \mathrm{~nm}$.

Starting from $\lambda_{1}=1552.2 \mathrm{~nm}$ for the first tap wavelength, the other wavelength are driven as $\lambda_{2}=1553.45 \mathrm{~nm}, \lambda_{3}=1554.7 \mathrm{~nm}$ and $\lambda_{4}=1555.95 \mathrm{~nm}$. To compensate the nonlinearity of dispersion effect, the actual wavelength corresponding the $2^{\text {nd }}$ and $3^{\text {rd }}$ taps, have to be practically a little different 1553.48 and $1554.67 \mathrm{~nm}$ respectively. The taps weights are $0 \mathrm{~dB}$ for the inner taps and $-12.8 \mathrm{~dB}$ for the outer.

To reference the system, a single carrier with $\lambda=1554.075 \mathrm{~nm}$ is used. This corresponds to a tap a zero time and can be though of as the $0^{\circ}$ arm of the hybrid.

\section{RESULTS}

To observe the $90^{\circ}$ phase shift, the VNA is calibrated setting the wavelength of LD1 to $\lambda=1554.075 \mathrm{~nm}$ and the other laser diodes turned off. The laser diodes were then all configured as described in Section III. Figure 4 presents the OSA trace indicating the relative wavelengths and amplitudes of the transversal filter. Figure 5 presents the measured frequency response from the VNA. The simulated response of Figure 3 is also presented in Figure 5 for comparison. Excellent agreement between the measurement and theory is evident. Amplitude variation $<3 \mathrm{~dB}$ has been maintained over the range from 2.517.5GHz, with negligible phase deviation from $90^{\circ}$.

\section{DISCUSSION}

The configuration of Figure 3 could be greatly simplified through the use of a balanced output modulator (with complimentary outputs). This would increase the link gain performance and reduce the component count. The optical combination could be achieved using wavelength multiplexers rather than simple $3 \mathrm{~dB}$ couplers. This will greatly improve the link gain $(6 \mathrm{~dB}$ electrical per split). Extension of this demonstration with these improvements is currently under investigation.

The tap weights in Figure 4 vary slightly from those depicted in Figure 1. This is due to the fact that different lasers with slightly different linewidths were used and the tap weight varies as the square of the total optical power.

The results presented in Figure 5 compare favorably traditional microwave hybrids. This photonic implementation offers the advantage that the pass band ripple and the bandwidth can dynamically adjusted changing the tap weights and/or the wavelength spacings.

It would be desirable to extend the hybrid operation to $2-40 \mathrm{GHz}$ with less than $3 \mathrm{~dB}$ ripple. To improve the bandwidth and ripple, extra taps could be introduced as discussed in [9]. Investigation of systems with additional wavelength taps is currently under investigation.

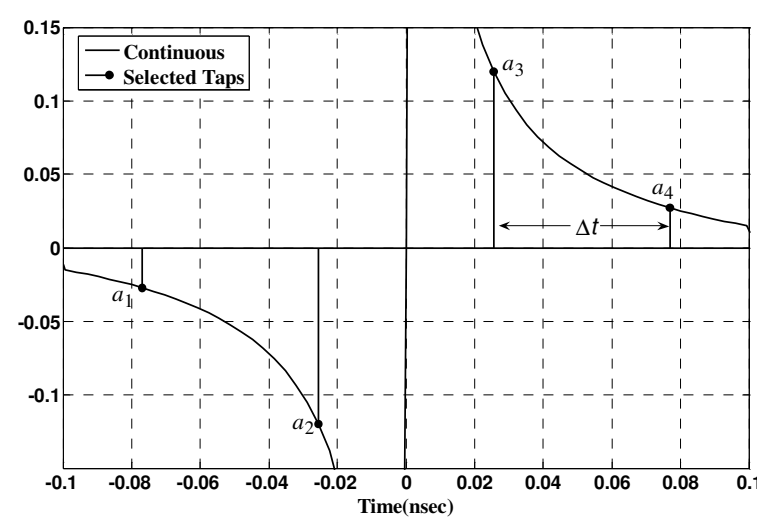

Fig. 1. Hyprebolic impulse response, continuous and infinite; and sampled.
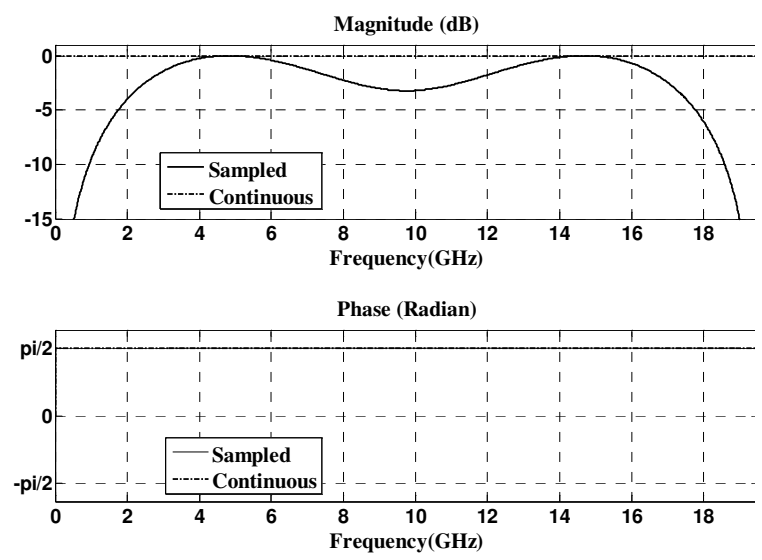

Fig. 2.Predicted frequency response for the impulse responses of Fig 1: continuous and sampled. 


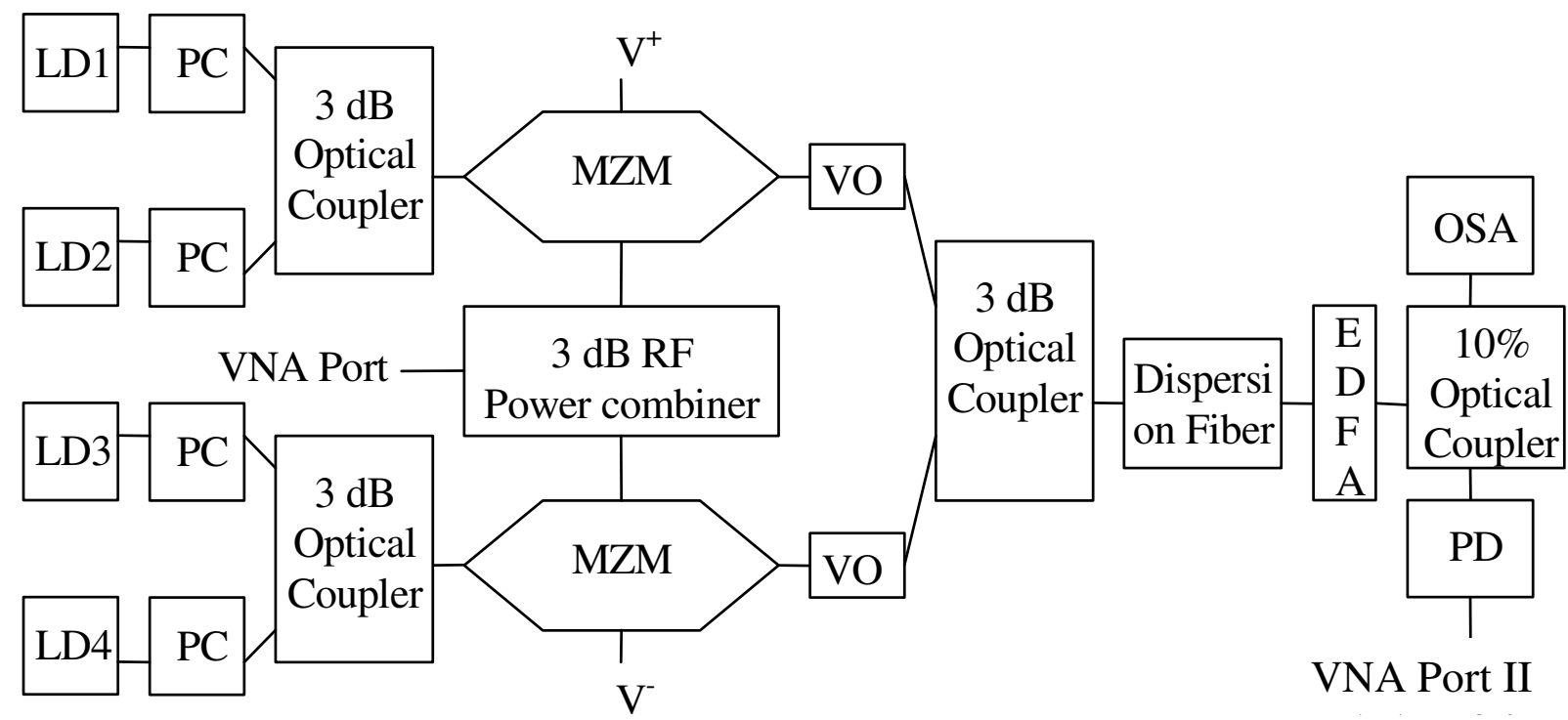

Figure 3: Block diagram of transversal filter experimental setup

\section{CONCLUSION}

A novel, photonic technique to realize a quadrature hybrid coupler using transversal scheme has been practically demonstrated. This photonic implementation offers a dynamically adjustable bandwidth and pass-band ripple. The demonstration can be readily extended to broader bandwidths through the introduction of extra wavelength channel taps.

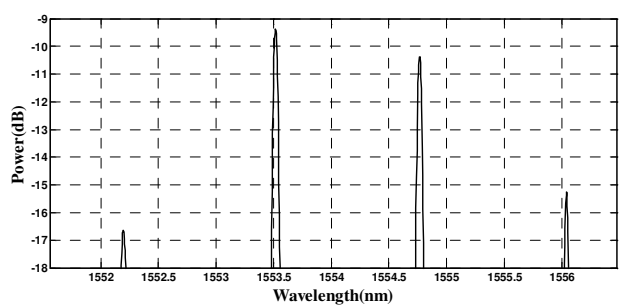

Fig. 4. OSA Trace indicating the relative weights and wavelengths of each tap.
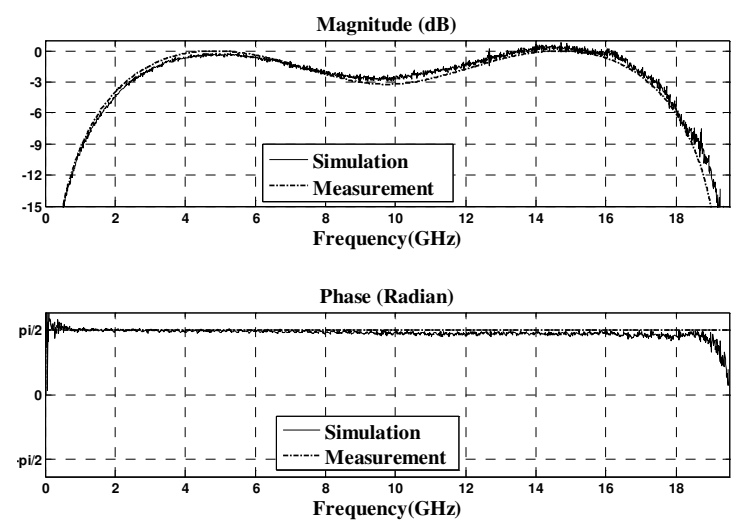

Fig. 5. a comparison between measurement and simulation result for both magnitude and phase

\section{REFERENCES}

[1] L. Fan, b. Heimer, and K. Chang, "Uniplanar hybrid couplers using asymmetrical coplanar strip lines," Microwave Symposium Digest, 1997, IEEE MTT-S International, 1997, pp. 273-276.

[2] R. T. Chen, E. R. Brown, R. S. Singh, "A compact $30 \mathrm{GHz}$ low loss balanced hybrid coupler fabricated using micromachined integrated coax," presented at Radio and Wireless conference, 2004 IEEE, pp. 227-230.

[3] J. S. Izadian, "A new 6-18 GHz, -3dB multisection hybrid coupler using asymmetric broadside, and edge coupled lines," IEEE Int. Microwave Symp, pp.143-246

[4] M. -H. <urgulescu, P. Leguad, E. Penard, and I. Zaquine, "New small $90^{\circ}$ hybrid coupler," Electronics Letters, vol. 30, pp. 1289-1290, 1994.

[5] G. A. Berenbrock and B. Schelmmer, "Active controlled fiber optical $90^{\circ}$ hybrid for coherent communications," IEEE Photon. Tech. Letters, vol.1, pp. 86-87, 1989.

[6] D. Madris, B. Vidal, A. Martinez, V. Polo, J. L. Corral, and J. Marti, "A new optical beamforming architecture based on $\mathrm{N} \times \mathrm{N}$ optical butler matrices providing $2 \mathrm{~N}$ beams," Antennas and Propagation Society International Symposium,2002. IEEE, 2002.

[7] S. Mansoori, A. Mitchell, K. Ghorbani "Photonic reconfigurable microwave filter with negative coefficients" Electronics Letters , vol. 40, pp 541-543, 2004.

[8] S. Mansoori, A. Mitchell, "RF transversal filter using an AOTF," IEEE Photonics Technology Letters, vol. 16, pp 879-881, Mar (2004).

[9] H. Emami, L. A. Bui, A. Mitchell, "Broadband 90 degree hybrid coupler using photonic transversal approach," APMWP 2006 